\title{
A Method for Optimal Analysis of Fields With Spatially Variable Mean
}

\author{
P. Y. LE TRAON
}

\author{
Collecte Localisation Satellites Argos, Toulouse, France
}

\begin{abstract}
This brief report addresses the problem of objective analysis of fields with spatially variable mean. Conventional objective analysis is confined to fluctuations, where the larger scale is assumed to be known; this report shows how to use the method described by Davis (1985) to estimate simultaneously a large-scale component and a mesoscale component with an accurate error budget. The extension to multivariate analysis is also discussed. The method is then applied to simulated data; this illustrates its contribution in relation to conventional objective analysis.
\end{abstract}

\section{INTRODUCTION}

Conventional objective analysis is confined to fluctuations, where the larger-scale component of the signal is assumed to be known and is subtracted from the data. In cases where the mean component is not perfectly known (the usual case) the estimator is generally no longer optimal, and the error maps do not take into account the additional error arising from difficulties in distinguishing between the mean field and the mesoscale fluctuations. However, a functional form of the mean field can usually be assumed, and this should be used in the analysis.

The technique below elaborates on this idea and gives a general framework for simultaneously estimating the mean and the fluctuation fields. This can be seen as a generalization of the objective analysis of a field with a constant mean as presented by Bretherton et al. [1976]. As in the work of Bretherton et al. [1976], our estimator will be a best (in a least squares sense) linear unbiased estimator which has the advantage of being a maximum likelihood estimator for Gaussian fields (in the other cases it has, however, no general optimum properties). Our method will closely follow the ideas presented by Davis [1985] for estimation of the large-scale component of the signal and will extend this approach to the multivariate case. The objective here is not necessarily to determine the large-scale component precisely but to incorporate it in the analysis and error budget. This large-scale component estimation is similar to the classical problem (see, for example, Kendall and Stuart [1977]) in time series analysis of trend or seasonal effect removal to deal with stationary statistics. However, in our case this estimation will be done simultaneously with the mesoscale fluctuation field estimation.

This kind of analysis can be of practical value for mapping fields with a nonnegligible large-scale component. In such cases the specification of the mean field has a significant influence upon the mapped fields, which should be reflected in the error budget. Watts et al. [1989] have shown, for example, that the mapping of the Gulf Stream thermal front is very sensitive to the choice of the functional form of the mean field.

In the following discussion this technique will first be presented in the univariate case and then be extended to the multivariate case. An application to simulated data will also be given to demonstrate the usefulness of the method. We

Copyright 1990 by the American Geophysical Union.

Paper number 90.JC01136.

$0148-0227 / 90 / 90 . J C-01136 \$ 02.00$ will assume, as in conventional objective analysis, that we have good a priori statistical knowledge of mesoscale fluctuations.

\section{Univariate Analysis}

\subsection{Simultaneous Data}

Let $\Psi_{\text {obs }}\left(\mathbf{x}_{i}\right)(i=1, \cdots, N)$ be the $N$ observations of a field $\Psi$ at a given time $t_{0}$ and at positions $\mathbf{x}_{i}=\left(x_{i}, y_{i}\right) . \Psi$ is assumed to consist of a large- (spatial) scale component and a mesoscale component: $\Psi(\mathrm{x})=\{\Psi(\mathrm{x})\}+\Psi^{\prime}(\mathrm{x})$. The largescale field is defined by the smoothing operator $\{\cdot\}$.

$\Psi_{\mathrm{obs}}\left(\mathrm{x}_{i}\right)=\Psi\left(\mathbf{x}_{i}\right)+\varepsilon_{\Psi}\left(\mathrm{x}_{i}\right)=\left\{\Psi\left(\mathbf{x}_{i}\right)\right\}+\Psi^{\prime}\left(\mathbf{x}_{i}\right)+\varepsilon_{\Psi}\left(\mathbf{x}_{i}\right)$

The noise $\varepsilon_{\Psi}\left(\mathbf{x}_{i}\right)$ is instrumental error and small unresolvable scales of $\Psi r . \Psi^{\prime}(\mathrm{x})$ is mesoscale turbulence.

The large-scale component is estimated using the method described by Davis [1985]. He selects a complete set of functions $F_{m}(\mathrm{x})$, so that any function $f$ of $\mathrm{x}$ can be expanded over the area of interest:

$$
f(\mathbf{x})=\sum_{m=1}^{\infty} b_{m} F_{m}(\mathbf{x})
$$

The functions $F_{m}(\mathrm{x})$ may be chosen from a complete set of orthogonal functions such as polynomial or Fourier series. Note, however, that the method does not require the functions $F_{m}(\mathrm{x})$ to be orthogonal.

The spatial filter $\{\cdot\}$ is then specified to pass the first $M$ functions while rejecting all others:

$$
\{\Psi(\mathbf{x})\}=\sum_{m=1}^{M} b_{m} F_{m}(\mathbf{x})
$$

i.e., the large-scale part is defined by the truncated sumiover the first $M$ basic functions, assumed to be ordered inversely to their spatial scales. These $M$ functions should preserve the large-scale features of the field and remove all the mesoscale features.

The field $\Psi(x)$ is estimated as a linear combination of the $N$ observations, and the weights are selected to minimize the mean square error as in conventional objective analysis:

$\Psi_{\text {est }}(\mathbf{x})=\sum_{n=1}^{N} \beta\left(\mathbf{x}, \mathbf{x}_{n}\right) \Psi_{\text {obs }}\left(\mathbf{x}_{n}\right)$ $e^{2}(x)=\left\langle\left[\Psi(\mathrm{x})-\Psi_{\text {est }}(\mathrm{x})\right]^{2}\right\rangle$ minimum 
where $\langle\cdot\rangle$ is an average over an ensemble of realizations such that

$$
\left\langle\Psi^{\prime}(\mathbf{x})\right\rangle=0 \quad\left\langle\varepsilon_{\Psi}\left(\mathbf{x}_{n}\right)\right\rangle=0 \quad \text { for any } \mathbf{x}, \mathbf{x}_{n}
$$

This means that the spatial filter is also a perfect filter for ensemble averaging, that is, we assume that the mean field (i.e., in the stochastic sense) has larger scales than the mesoscales.

The difference relative to objective analysis lies in the zero-bias constraint on the estimator:

$$
\left\langle\Psi_{\mathrm{est}}(\mathbf{x})-\Psi(\mathbf{x})\right\rangle=0
$$

which yields

$$
\sum_{m=1}^{M}\left\langle b_{m}\right\rangle\left[\sum_{n=1}^{N} \beta\left(\mathbf{x}, \mathbf{x}_{n}\right) F_{m}\left(\mathbf{x}_{n}\right)-F_{m}(\mathbf{x})\right]=0
$$

Following Davis [1985], we will force the analysis to pass the $M$ fundamental functions $F_{m}$ with unit gain:

$$
\sum_{n=1}^{N} \beta\left(\mathbf{x}, \mathbf{x}_{n}\right) F_{m}\left(\mathbf{x}_{n}\right)=F_{m}(\mathbf{x}) \quad m=1, \cdots, M
$$

With these conditions the zero-bias constraint is automatically fulfilled. The mean square error can then be written as

$$
\begin{aligned}
e^{2}(\mathbf{x})=C_{\mathrm{xx}}-2 \sum_{s=1}^{N} \beta\left(\mathrm{x}, \mathrm{x}_{n}\right) C_{\mathrm{x} n} & \\
& \quad+\sum_{r=1}^{N} \sum_{s=1}^{N} \beta\left(\mathrm{x}, \mathrm{x}_{r}\right) \beta\left(\mathbf{x}, \mathrm{x}_{s}\right) A_{r s}
\end{aligned}
$$

with

$$
\begin{aligned}
C_{\mathrm{x} n}= & \left\langle\Psi^{\prime}(\mathbf{x}) \Psi_{\mathrm{obs}}^{\prime}\left(\mathbf{x}_{n}\right)\right\rangle=\left\langle\Psi^{\prime}(\mathbf{x}) \Psi^{\prime}\left(\mathbf{x}_{n}\right)\right\rangle \\
A_{r s}= & \left\langle\Psi_{\mathrm{obs}}^{\prime}\left(\mathbf{x}_{r}\right) \Psi_{\mathrm{obs}}^{\prime}\left(\mathbf{x}_{s}\right)\right\rangle=\left\langle\Psi^{\prime}\left(\mathbf{x}_{r}\right) \Psi^{\prime}\left(\mathbf{x}_{s}\right)\right\rangle \\
& +\left\langle\varepsilon_{\Psi}\left(\mathbf{x}_{r}\right) \varepsilon_{\Psi}\left(\mathbf{x}_{s}\right)\right\rangle
\end{aligned}
$$

In these expressions $\Psi_{\text {obs }}^{\prime}\left(\mathbf{x}_{r}\right)$ is defined as $\Psi_{\text {obs }}\left(\mathbf{x}_{r}\right)$ $\left\{\Psi\left(\mathbf{x}_{r}\right)\right\}=\Psi^{\prime}\left(\mathbf{x}_{r}\right)+\varepsilon_{\Psi}\left(\mathbf{x}_{r}\right)$.

The minimization problem is solved using Lagrange multipliers. The result is

$$
\begin{aligned}
\beta\left(\mathbf{x}, \mathbf{x}_{n}\right)= & \sum_{m=1}^{M}\left[F_{m}(\mathbf{x})-G_{m}(\mathbf{x})\right] \\
& \sum_{k=1}^{M} Z_{m k}^{-1} \sum_{p=1}^{N} A_{n p}^{-1} F_{k}\left(\mathbf{x}_{p}\right)+\sum_{i=1}^{N} A_{n i}^{-1} C_{\mathbf{x} i}
\end{aligned}
$$

where

$$
\begin{aligned}
& Z_{m k}=\sum_{r=1}^{N} \sum_{s=1}^{N} A_{r s}^{-1} F_{m}\left(\mathbf{x}_{r}\right) F_{k}\left(\mathbf{x}_{s}\right) \\
& G_{m}(\mathbf{x})=\sum_{r=1}^{N} F_{m}\left(\mathbf{x}_{r}\right) \sum_{s=1}^{N} A_{r s}^{-1} C_{\mathrm{x} s}
\end{aligned}
$$

which in turn yields

$$
\begin{aligned}
\Psi_{\text {est }}(\mathbf{x})= & \sum_{m=1}^{M} F_{m}(\mathbf{x}) \sum_{k=1}^{M} Z_{m k}^{-1} \sum_{p=1}^{N} \sum_{n=1}^{N} A_{n p}^{-1} F_{k}\left(\mathbf{x}_{p}\right) \Psi_{\text {obs }}\left(\mathbf{x}_{n}\right) \\
& +\sum_{r=1}^{N} \sum_{s=1}^{N} A_{r s}^{-1} C_{\mathrm{x} s}\left[\Psi_{\mathrm{obs}}\left(\mathbf{x}_{r}\right)-\sum_{m=1}^{M} F_{m}\left(\mathbf{x}_{r}\right)\right. \\
& \left.\cdot \sum_{k=1}^{M} Z_{m k}^{-1} \sum_{p=1}^{N} \sum_{n=1}^{N} A_{m p}^{-1} F_{k}\left(\mathbf{x}_{p}\right) \Psi_{\text {obs }}\left(\mathbf{x}_{n}\right)\right]
\end{aligned}
$$

This is similar to the result found by Bretherton et al. [1976] for a constant estimated mean $\left(M=1, F_{1}(\mathrm{x})=1\right)$. This also can be compared to the result of optimal analysis of a field with a spatially variable, but perfectly known, mean. In the latter case it can indeed be easily shown [e.g., Bretherton and McWilliams, 1980] that the best linear unbiased estimator at $\mathrm{x}$ is given by

$$
\Psi_{\mathrm{est}}(\mathbf{x})=\langle\Psi(\mathbf{x})\rangle+\sum_{r=1}^{N} \sum_{s=1}^{N} A_{r s}^{-1} C_{\mathrm{x} s}\left[\Psi_{\mathrm{obs}}\left(\mathbf{x}_{r}\right)-\left\langle\Psi\left(\mathbf{x}_{r}\right)\right\rangle\right]
$$

The mean field $\langle\Psi(\mathbf{x})\rangle$ is subtracted from each observation value $\Psi_{\text {obs }}\left(\mathbf{x}_{r}\right)$ and added back to the estimate. This is similar to $(7 a)$, where the mean field was not assumed to be known a priori. However, the mean field in (7b) is replaced in (7a) by a large-scale estimated field $\{\Psi(\mathbf{x})\}$ which is not necessarily stationary (in the stochastic sense). The large-scale component is that found by Davis [1985]. By estimating simultaneously the large-scale component and the mesoscale component, it is possible, however, to take account of the error due to the unknown mean.

The effect of not knowing the mean flow reappears indeed in the associated error calculation. We thus have

$$
\begin{aligned}
e^{2}(\mathbf{x})= & C_{\mathrm{xx}}-\sum_{r=1}^{N} \sum_{s=1}^{N} C_{\mathrm{x} r} C_{\mathrm{x} s} A_{r s}^{-1} \\
& +\sum_{m=1}^{M} \sum_{k=1}^{M} Z_{m k}^{-1}\left[F_{m}(\mathrm{x})-G_{m}(\mathrm{x})\right]\left[F_{k}(\mathrm{x})-G_{k}(\mathrm{x})\right]
\end{aligned}
$$

The last term is the additional error due to uncertainty on the mean field, which is, of course, different from the error on the mean field only.

\subsection{Nonsimultaneous Data}

Generally, data gathered at different time periods are used, particularly since this provides a better description of the mean field. For example, one can take $N$ measurements at time $t_{0}$ and points $\mathbf{x}_{n}$, which are thus correlated with $\Psi^{\prime}(\mathbf{x}$, $t_{0}$ ), and $M$ additional measurements at other times $t_{m}$ which may not be correlated with $\Psi^{\prime}\left(\mathrm{x}, t_{0}\right)$ if $\left[t_{m}-t_{0}\right]>T_{d}$, where $T_{d}$ is the mesoscale decorrelation time. However, these measurements are still useful for the estimation of the large-scale field since $T_{\{\cdot\}} \gg T_{d}$ where $T_{\{\cdot\}}$ is a characteristic time scale of the large-scale field. In this case the correlation matrix takes the form 


$$
\left[A_{r s}\right]=\left[\begin{array}{cc}
A_{0} & (0) \\
(0) & A_{m}
\end{array}\right]
$$

and

$$
\left[A_{r s}^{-1}\right]=\left[\begin{array}{cc}
A_{0}^{-1} & (0) \\
(0) & A_{m}^{-1}
\end{array}\right]
$$

From (7) it can be seen that all the terms of the inverse matrix appear in the calculation of the mean field, while only the $A_{0}^{-1}$ term appears in the expression for the fluctuating part.

Using nonsimultaneous data makes it easier to distinguish between the mean field and the mesoscale field, as the two have different temporal scales. The constraint of having a $\{\cdot\}$ field with a larger spatial scale than the mesoscale can even be relaxed if enough data are used to permit a separation in time, rather than a separation in space.

\section{Multivariate Analysis}

This approach can be extended to observations which may not be direct observations of the $\Psi$ field to be estimated. For example, let $u$ and $v$ be two variables such that $u=L_{1}(\Psi)$ and $v=L_{2}(\Psi)$, where $L_{1}$ and $L_{2}$ are linear differential or integral operators. $\Psi$ will thus be estimated as a linear combination of $u_{\mathrm{obs}}\left(\mathbf{x}_{i}\right)$ and $v_{\mathrm{obs}}\left(\mathbf{x}_{j}\right)$, i.e.,

$$
\Psi_{\mathrm{est}}(\mathbf{x})=\sum_{i=1}^{N 1} \alpha\left(\mathbf{x}, \mathbf{x}_{i}\right) u_{\mathrm{obs}}\left(\mathbf{x}_{i}\right)+\sum_{j=1}^{N 2} \beta\left(\mathbf{x}, \mathbf{x}_{j}\right) v_{\mathrm{obs}}\left(\mathbf{x}_{j}\right)
$$

The zero-bias constraint yields

$$
\begin{aligned}
\sum_{m=1}^{M}\left\langle b_{m}\right\rangle F_{m}(\mathbf{x})= & \sum_{i=1}^{N 1} \alpha\left(\mathbf{x}, \mathbf{x}_{i}\right)\left\langle u_{\text {obs }}\left(\mathbf{x}_{i}\right)\right\rangle \\
& +\sum_{j=1}^{N 2} \beta\left(\mathbf{x}, \mathbf{x}_{j}\right)\left\langle v_{\text {obs }}\left(\mathbf{x}_{j}\right)\right\rangle \\
= & \sum_{i=1}^{N 1} \alpha\left(\mathbf{x}, \mathbf{x}_{i}\right)\left\langle L_{1}\left[\Psi\left(\mathbf{x}_{i}\right)\right]+\varepsilon_{u}\left(\mathbf{x}_{i}\right)\right\rangle \\
& +\sum_{j=1}^{N 2} \beta\left(\mathbf{x}, \mathbf{x}_{j}\right)\left\langle L_{2}\left[\Psi\left(\mathbf{x}_{j}\right)\right]+\varepsilon_{u}\left(\mathbf{x}_{j}\right)\right\rangle
\end{aligned}
$$

but

$$
\begin{aligned}
& \left\langle L_{1}\left[\Psi\left(\mathbf{x}_{i}\right)\right]+\varepsilon_{u}\left(\mathbf{x}_{i}\right)\right\rangle=\left\langle L_{1}\left[\Psi\left(\mathbf{x}_{i}\right)\right]\right\rangle \\
& =L_{1}\left[\left\langle\left\{\Psi\left(\mathbf{x}_{i}\right)\right\}\right\rangle\right]=\sum_{m=1}^{M}\left\langle b_{m}\right\rangle L_{1}\left[F_{m}\left(\mathbf{x}_{i}\right)\right]
\end{aligned}
$$

since $L_{1}(0)=0$ and the operators $L_{1}$ and $\langle\cdot\rangle$ are assumed to commute. There is a similar expression for $L_{2}$.

This shows that if

$$
F_{m}(\mathbf{x})=\sum_{i=1}^{N 1} \alpha\left(\mathbf{x}, \mathbf{x}_{i}\right) L_{1}\left[F_{m}\left(\mathbf{x}_{i}\right)\right]
$$

$$
+\sum_{j=1}^{N 2} \beta\left(\mathbf{x}, \mathbf{x}_{j}\right) L_{2}\left[F_{m}\left(\mathbf{x}_{j}\right)\right] \quad m=1, \cdots, M
$$

then the zero-bias condition is fulfilled.

Taking $\Psi_{\mathrm{obs}}\left(\mathrm{x}_{i}\right)=u_{\mathrm{obs}}\left(\mathrm{x}_{i}\right)(i=1, \cdots, N 1)$ and $\Psi_{\mathrm{obs}}\left(\mathbf{x}_{i}\right)=$ $v_{\text {obs }}\left(\mathbf{x}_{i}\right)(i=N 1+1, \cdots, N 1+N 2)$ and considering the correlations between the variables $\Psi_{\text {obs }}^{\prime}\left(\mathrm{x}_{i}\right)=\Psi_{\text {obs }}\left(\mathrm{x}_{i}\right)-$ $L\{\Psi\}$ alone in matrix $A$ and with $\Psi^{\prime}(\mathbf{x})$ in the $C$ terms, the same formalism as above can be applied (equation (7)). However, the $F_{m}\left(\mathrm{x}_{p}\right)$ must be replaced by $L_{1}\left[F_{m}\left(\mathrm{x}_{p}\right)\right]$ for $p$ $=1, \cdots, N 1$ or $L_{2}\left[F_{m}\left(\mathrm{x}_{p}\right)\right]$ for $p=N 1+1, \cdots, N 1+N 2$.

It should also be noted that the correlations among $\Psi$ ', $u_{\text {obs }}^{\prime}, v_{\text {obs }}^{\prime}$ can also be obtained analytically by knowing $\left\langle\Psi^{\prime}\left(\mathrm{x}_{1}\right) \Psi^{\prime}\left(\mathrm{x}_{s}\right)\right\rangle$, given that $L_{1}$ and $L_{2}$ are linear operators.

\section{Application}

P. Y. Le Traon and M. Ollitrault (Description of an eddy west of the Mid Atlantic Ridge near $36^{\circ} \mathrm{N}, 40^{\circ} \mathrm{W}$, submitted to Journal of Marine Research (1990); hereinafter Le Traon and Ollitrault (submitted manuscript, 1990)) have used this multivariate analysis on velocities and dynamic height measurements to describe the dynamic height field at $100 \mathrm{dbar}$ in a $120-\mathrm{km}$ latitude, $150-\mathrm{km}$ longitude area near $36^{\circ} \mathrm{N}, 40^{\circ} \mathrm{W}$. This was a simple case in which three fundamental functions were chosen to describe the large-scale field: $F_{1}(\mathbf{x})=1$, $F_{2}(\mathrm{x})=y$, and $F_{3}(\mathrm{x})=x$ with $\mathrm{x}=(x, y)$. Mesoscale autocorrelation functions of sea surface height were derived from satellite altimetry, and the large-scale component was introduced to deal with a possible mean field (Azores current) or larger variability scales not taken into account in satellite altimetry covariance functions. We showed that this large-scale field and the mesoscale field can be estimated simultaneously. However, in this particular case only a slightly better mapping of the field is obtained because the mean field is weak in relation to the mesoscale field (see Le Traon and Ollitrault (submitted manuscript, 1990) for a more detailed discussion).

This brief report provides a more convincing example which demonstrates the usefulness of the method. A dynamic height field $D(\mathrm{x}), \mathrm{x}=(x, y)$ on a 500 - by $500-\mathrm{km}$ grid and a $25-\mathrm{km}$ ( $x$ and $y$ ) sampling interval, was simulated using a Gaussian pseudorandom vector generator. The field is determined completely by the mean value $\langle D(\mathrm{x})\rangle$ at each point and the two-point covariances $\left\langle D\left(\mathrm{x}_{1}\right) D\left(\mathrm{x}_{2}\right)\right.$ for all $\mathrm{x}_{1}$, $\mathbf{x}_{2}$ pairs. These parameters were chosen as follows (where $D$ is in centimeters and $y$ is in kilometers):

$$
\begin{gathered}
\langle D(\mathrm{x})\rangle=20-100(y / 500)^{2} \\
\left\langle D\left(\mathrm{x}_{1}\right) D\left(\mathrm{x}_{2}\right)\right\rangle=\sigma^{2}\left[1+a r+\frac{1}{6}(a r)^{2}-\frac{1}{6}(a r)^{3}\right] e^{-a r} \\
\left.r=\left[x_{1}-x_{2}\right)^{2}+\left(y_{1}-y_{2}\right)^{2}\right]^{1 / 2} \\
a=50 \mathrm{~km}^{-1} \quad \sigma=20 \mathrm{~cm}
\end{gathered}
$$

The mean field amplitude varies between $20 \mathrm{~cm}$ and -80 $\mathrm{cm}$. Such a range is typical of the variations observed in western boundary currents. The covariances were obtained from the analytical model put forward by Arhan and Colin de Verdière [1985], assuming isotropic, homogeneous, mesoscale turbulence.

Figure 1 shows a simulated dynamic height field, i.e., a 


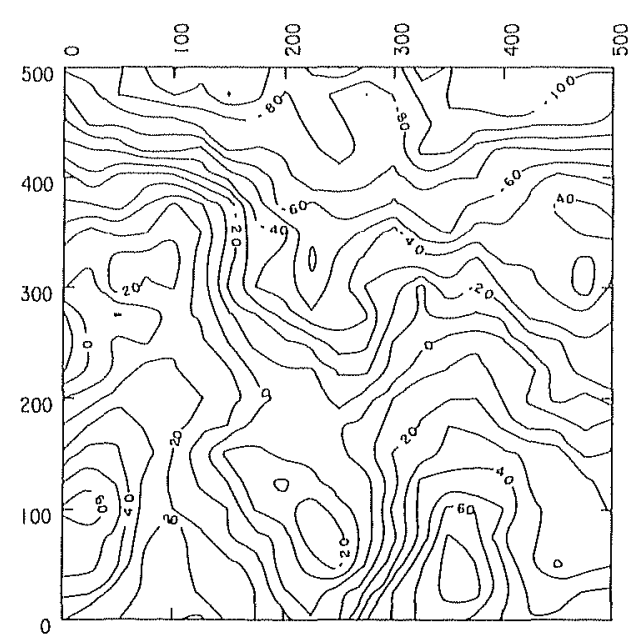

Fig. 1. Simulated reference dynamic height field (units are centimeters) on a $500-\mathrm{km}$ by $500-\mathrm{km}$ grid. It is a particular realization of a Gaussian field $D(\mathrm{x})$, with $\mathrm{x}=(x, y)$, demonstrating the following statistical properties: $\langle D(\mathrm{x})\rangle=20-100(y / 500)^{2}(D$ in centimeters, $y$ in kilometers) and $\left\langle D\left(\mathbf{x}_{1}\right) D\left(\mathbf{x}_{2}\right)\right\rangle=\sigma^{2}\left[1+a r+\frac{1}{6}(a r)^{2}-\right.$ $\left.\frac{1}{6}(a r)^{3}\right] e^{-a r}$ with $r=\left[\left(x_{1}-x_{2}\right)^{2}+\left(y_{1}-y_{2}\right)^{2}\right]^{1 / 2}, a=50 \mathrm{~km}^{-1}$, and $\sigma=20 \mathrm{~cm}$.

particular realization of the statistical set defined above. We shall take this as the reference field. Sixteen simulated measurements were selected from the field. A random Gaussian error with zero mean and a variance of $20 \mathrm{~cm}^{2}(5 \%$ of the variance of the mesoscale field) was added to each such measurement. The method described above was then applied, where the mean field was assumed to have the following functional form: $\langle D(\mathrm{x})\rangle=b_{1}+b_{2} y+b_{3} y^{2}$ (it is not assumed in advance that $b_{2}$ is zero). The field obtained is shown in Figure $2 a$. It can be compared to the field which would be obtained by conventional objective analysis (subtraction of the data mean, analysis of fuctuations, and addition of the data mean to the estimated fluctuation field) (Figure $2 b$ ).

Although the relatively loose sampling does not permit an accurate description of the small structures shown in Figure

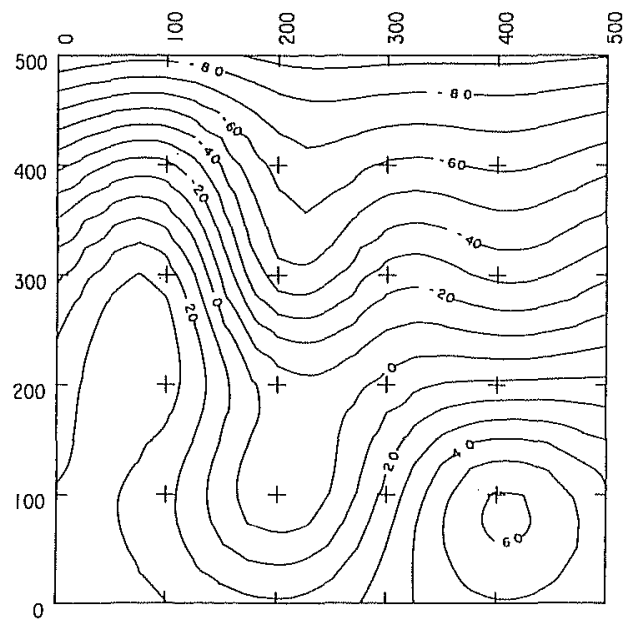

Fig. 2a. Reconstructed dynamic height field using the method described in this brief report (units are centimeters). A random noise of $20 \mathrm{~cm}^{2}$ was added to each of the 16 data measurements (plus signs) before applying the method. The main features of the reference field are well reconstructed.

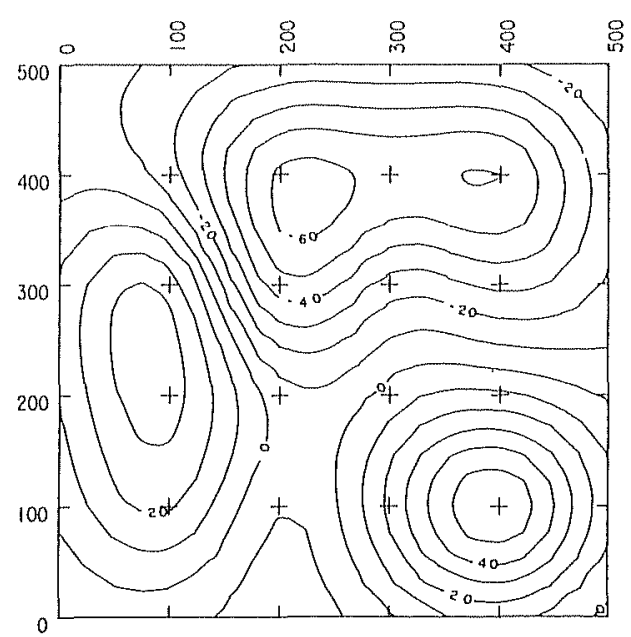

Fig. $2 b$. Same as Figure $2 a$, but using conventional objective analysis, i.e., by removing, before analysis, a constant mean, analyzing the fluctuations, and adding back the constant estimated mean. This kind of analysis does not provide a good estimation in areas far from the data measurements.

1, the expected general shape is observed. However, this shape is not observed in conventional objective analysis, in particular close to the edges (Figure $2 b$ ). In the center of the area the analyses are fairly similar, as they are significantly constrained by the data. The error maps for our analysis (Figure $3 a$ ) and conventional objective analysis (Figure $3 b$ ) also differ and provide a means of estimating the additional error due to lack of knowledge of the mean field (the last term of (8)). It can be seen that this error increases considerably in areas far from the data measurements. This error is typically $1-2 \mathrm{~cm}$ at the center of the area and reaches a maximum of $15 \mathrm{~cm}$ at the edges. The error map given by conventional objective analysis, which does not take account of the term, is not at all realistic, and the estimated errors are considerably lower than the differences between the field estimated in Figure $2 b$ and the reference field.

In conclusion, we have demonstrated that it is possible to estimate, simultaneously, a mean field and a mesoscale fluctuation field. In particular, we have shown that the

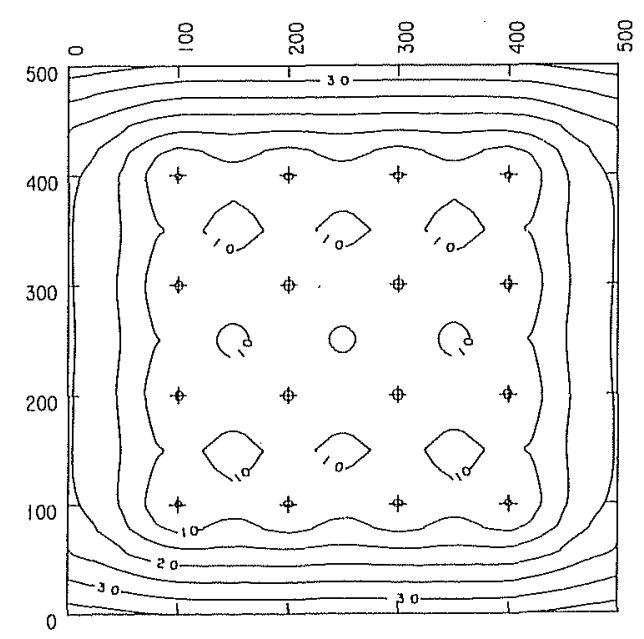

Fig. $3 a$. Error map in centimeters corresponding to Figure $2 a$. The additional error due to the unknown mean field is typically $1-2$ $\mathrm{cm}$ in the center of the area but can reach $15 \mathrm{~cm}$ at the edges. 


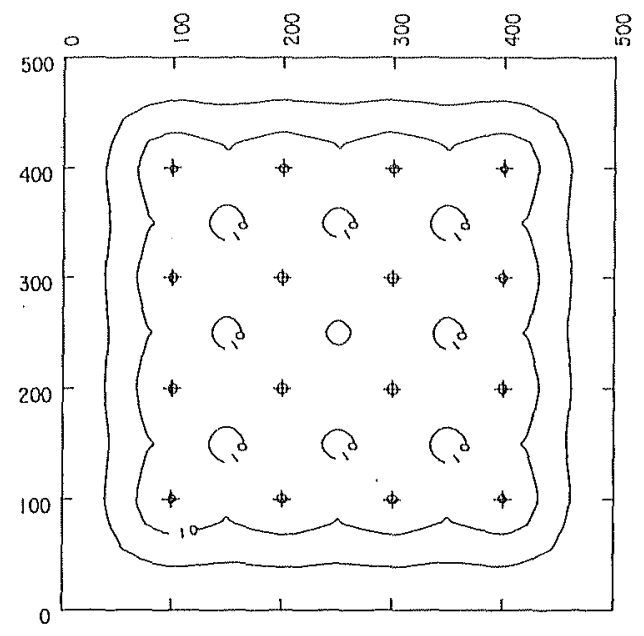

Fig. 3b. Error map in centimeters corresponding to Figure $2 b$ (conventional objective analysis). This error map is not realistic, since it assumes the mean field is perfectly known and thus largely underestimates the error field.

additional error due to lack of knowledge of the mean field can also be estimated. This method, which is a simple generalization of conventional objective analysis, should be recommended for mapping the field when a significant mean component is present.
Acknowledgments. We thank M. Ollitrault, P. De Mey, and J. F. Minster for their helpful comments. This work was supported by CNRS/DRET contribution 509291 and an ARGOS CIFRE grant.

\section{REFERENCES}

Arhan, M., and A. Colin de Verdière, Dynamics of eddy motions in the eastern North Atlantic, J. Phys. Oceanogr., 15, 153-170, 1985.

Bretherton, F. P., and J. C. McWilliams, Estimations from irregular arrays, Rev. Geophys. 18(4), 789-8I2, 1980.

Bretherton, F. P., R. E. Davis, and C. B. Fandry, A technique for objective analysis design of oceanographic experiments applied to mode 73, Deep Sea Res., 23, 559-582, 1976.

Davis, R. E., Objective mapping by least square fitting, $J$. Geophys. Res., 90(C3), 4773-4777, 1985.

Kendall, M., and A. Stuart, The Advanced Theory of Statistics, 2 vols., MacMillan, New York, 1977.

Watts, D. R., K. L. Tracey, and A. I. Friedlander, Producing accurate maps of the Gulf Stream thermal front using objective analysis, $J$. Geophys. Res., 94(C6), 8040-8052, 1989.

P. Y. Le Traon, Collecte Localisation Satellites Argos, 18 Avenue Edouard Belin, 31055 Toulouse Cedex, France.

(Received October 4, 1989;

accepted December 6, 1989.) 